\title{
Estudios sobre Meteorización de Huesos en Patagonia
}

\author{
Studies on Bone Weathering in Patagonia
}

\section{Isabel Cruz ${ }^{\mathrm{i}}$}

\section{RESUMEN}

La meteorización es uno de los principales procesos que causan la destrucción de huesos en la superficie terrestre. En Patagonia, debido a la baja intensidad de la acción de los carnívoros, especialmente sobre restos de grandes vertebrados, la meteorización tiene un papel central en su deterioro. En este trabajo se presenta una síntesis de las investigaciones efectuadas en varios ambientes patagónicos, con el fin de evaluar qué es lo que conocemos y qué es lo que todavía es necesario investigar para que la meteorización se convierta en una adecuada herramienta para comprender la historia tafonómica de un depósito particular.

Palabras clave: Meteorización Ósea, Tafonomía de Vertebrados, Patagonia.

ABSTRACT

Weathering is one of the major processes that cause the destruction of bones on the earth's surface. In Patagonia, due to the low intensity of carnivores' action, especially on large vertebrate remains, weathering has a central role.The aim of this paper is to present a synthesis of the results obtained in several habitats of the region, in order to assess what we know and what we still need to study about this taphonomic process.

Key words: Bone Weathering, Vertebrate Taphonomy, Patagonia. 


\section{INTRODUCCIÓN}

La meteorización es el proceso por el cual los componentes orgánicos e inorgánicos del hueso se separan unos de otros y se destruyen por la acción de los agentes físicos y químicos que operan sobre el hueso, tanto en la superficie como dentro del suelo (Behrensmeyer 1978). Desde el punto de vista tafonómico, este proceso es uno de los más importantes entre los que destruyen huesos sobre la superficie de la tierra (Lyman 1994). En el marco de la zooarqueología, los patrones de meteorización se utilizan para evaluar el grado de exposición de los huesos antes de ser cubiertos por sedimentos, para estimar la mezcla de huesos de diferentes edades y orígenes y como una medida del grado de conservación de depósitos arqueológicos, entre algunos ejemplos.

Gran parte del conocimiento que poseemos sobre la meteorización proviene de estudios que buscaron comprender la relación entre variables ambientales y sus consecuencias sobre los restos orgánicos a través de observaciones actuales en diferentes regiones. Entre estos estudios se destacan los desarrollados por zooarqueólogos patagónicos en relación a huesos de varios vertebrados (Belardi 1999, Borella 2004, Borrero 1990, 2007; Cruz 2007a, 2008; Muñoz y Savanti 1998; entre otros). El interés de estas investigaciones, más allá del que puedan tener para los estudios regionales, radica en que fueron realizadas en contextos diferentes a los involucrados en los estudios clásicos sobre este proceso. Patagonia presenta condiciones climáticas y ecológicas particulares que, desde el punto de vista tafonómico, la diferencian claramente de los ambientes en que dichos estudios se desarrollaron (Borrero 2000, 2007; Cruz 2008, 201I; entre otros). En esta región el rol de la meteorización es fundamental debido a la escasa importancia de la acción de carnívoros, especialmente sobre restos de grandes vertebrados (Borrero 1990, 2007). Como las tasas de meteorización varían en función de variables climáticas y ambientales, de las características esqueletarias y el tamaño de los taxones involucrados (Behrensmeyer 1978; Borrero 2007; entre otros), es necesario efectuar estudios regionales que permitan comprender adecuadamente las posibles variaciones entre ambientes y taxones. De esta manera, la evaluación de la meteorización en los conjuntos zooarqueológicos podrá ser una herramienta heurística para comprender la formación de los depósitos.

En este marco, el objetivo de este trabajo es sintetizar las investigaciones sobre el tema efectuadas en Patagonia hasta el momento, enfatizando el grado de conocimiento alcanzado para huesos de vertebrados de la región y la comparación entre taxones.

\section{ASPECTOS METODOLÓGICOS}

Todas las investigaciones que se mencionan son observaciones actuales que se abordaron en escalas espaciales amplias y partieron de un enfoque naturalista. Esto último implica que no se realizaron en laboratorios o ambientes controlados sino que se centraron en huesos depositados naturalmente. Tanto la escala como este enfoque permiten registrar la diversidad y la regularidad que caracterizan a los procesos tafonómicos en función de las condiciones imperantes en distintos contextos (Cruz 2009).

Tal como efectuara Beherensmeyer (1978), algunos de estos estudios evaluaron la meteorización a partir de transectas en diversos hábitats, estableciendo las variaciones en el espacio. Este es el caso de las investigaciones de Muñoz y Savanti (1998), Belardi (1999), Borella (2004) y Cruz (2007a, 2008, 2009; entre otros). Otros efectuaron observaciones a largo plazo, como Borrero (2007) y Cruz y Muñoz (20l0, 20I I), registrando los cambios a través del tiempo.

Los estudios se efectuaron en hábitats de la estepa patagónica, su ecotono con el bosque y la costa atlántica, abarcando un amplio espectro ambiental de la región. Muchos de los resultados obtenidos son concordantes con los de otros lugares del mundo, pero también permitieron establecer la existencia de diferencias, mostrando que, como en todo proceso tafonómico, los factores ambientales y ecológicos son de gran importancia al considerar la meteorización.

\section{LAS OBSERVACIONES SOBRE METEORIZACIÓN}

Guanaco (Lama guanicoe)

Si bien a lo largo de estas últimas décadas ha habido diversos estudios tafonómicos dedicados a 
esta especie, las investigaciones sobre meteorización no son muchas. Las primeras observaciones fueron efectuadas por Borrero (1990, entre otros) en Tierra del Fuego, en el marco de estudios tafonómicos más amplios en el ecotono bosque-estepa. Sus estudios se basaron en trece carcasas, que fueron repetidamente visitadas a lo largo de dieciséis años. Para evaluar la meteorización, utilizó los estadios propuestos por Behrensmeyer, diseñados para estimar este proceso en mamíferos terrestres de más de $5 \mathrm{~kg}$.

Borrero señala la gran importancia de la meteorización en el reciclado de huesos de guanaco en el sur del continente, lo cual no sucede con los huesos de ungulados en ambientes como los africanos, donde los carnívoros tienen el papel principal.Además, estima las tasas de meteorización en Tierra del Fuego, confirmando que existen diferencias entre los distintos elementos del esqueleto: partes del cráneo, la hoja de la escápula, vértebras y costillas meteorizan más rápidamente que los huesos largos (Borrero 2007).

También nota que las tasas varían entre hábitats, ya que tal como fuera detectado por Tappen (1994) son más lentas en el bosque, donde la vegetación tiene un rol importante mitigando los efectos de los agentes atmosféricos. Un aspecto interesante es que, contrariamente a lo esperado, las tasas de meteorización estimadas son más rápidas que las comunicadas por Behrensmeyer (1978) para Amboseli. Entre los factores responsables de la rapidez de este proceso, Borrero menciona los ciclos de congelamiento-descongelamiento que periódicamente afectan a los huesos.

En un ambiente más seco y templado en Patagonia continental, Cruz y Muñoz (20l0, 20II) estudian la meteorización en huesos de diversos vertebrados a través de observaciones a largo plazo. En el caso de los guanacos, las observaciones incluyeron cinco carcasas que se controlaron dos veces por año desde 2005. Debido a que es un proyecto todavía en curso, sólo se estimaron las tasas correspondientes a los estadios 0,1 y 2 , que son más lentas que las determinadas para estos estadios por Behrensmeyer en África y Borrero en Tierra del Fuego. Otro aspecto registrado es que los huesos largos de guanaco meteorizan más rápidamente que los huesos compactos (como los de las articulaciones de muñecas y tobillos) y que las superficies planas de mandíbulas, pelvis, costillas y vértebras.

\section{Cetáceos}

Otro taxón presente en depósitos arqueológicos del sur del continente son los cetáceos, vinculados a la explotación de animales varados en sectores costeros. Borella (2004) propuso que, en función de las características histológicas de los cetáceos, no es posible utilizar los estadios definidos por Behrensmeyer. Considerando este aspecto, avanzó en la determinación del patrón de meteorización de sus huesos en la región. Notó que, a diferencia de lo que ocurre con los huesos de mamíferos terrestres, en los que las grietas de meteorización se ubican longitudinalmente con respecto al eje mayor del hueso, en los huesos de cetáceos estas grietas presentan un patrón poligonal. Paralelamente a la aparición de las grietas comienza la exfoliación de las capas externas del hueso que en los cetáceos son muy delgadas, exponiendo así el tejido esponjoso. Al avanzar el proceso de meteorización, las grietas se profundizan y el hueso se fragmenta en fracciones amorfas hasta el colapso total. Este patrón fue observado en cuerpos vertebrales y huesos largos (Borella 2004). Otro aspecto registrado es que existen diferencias en las tasas de meteorización de huesos fusionados y no fusionados, ya que estos últimos meteorizan más rápidamente.

\section{Lobo marino (Otaria flavescens)}

Los huesos de lobos marinos son muy comunes en registros arqueológicos de la costa de Patagonia y del resto del continente. Aunque se han desarrollado estudios tafonómicos en diversos sectores de la región, son pocos los resultados sobre la forma en que meteorizan los huesos de estos mamíferos.

Borella y Muñoz (2006) plantearon que los perfiles de meteorización de huesos actuales de pinnípedos en la costa de Tierra del Fuego presentan una gran diversidad de estadios, mientras que los de conjuntos arqueológicos de la región son más homogéneos. Según estos autores, esto permite diferenciar ambos tipos de depósitos. Considero que este es un indicador ambiguo, ya que los perfiles de meteorización dependen de la historia de formación de los depósitos y no del agente de depositación.

Los estudios desarrollados en Patagonia continental evaluaron la meteorización a partir 
de la observación de cuatro carcasas y dos concentraciones de huesos de Otaria flavescens (Cruz y Muñoz 20II). Se estableció que los estadios propuestos por Behrensmeyer no son totalmente adecuados para evaluar la meteorización de los huesos de estos mamíferos, por lo que en el futuro habrá que precisar el patrón específico para cada estadio.

Entre los resultados obtenidos hasta el momento se cuentan las tasas correspondientes a los estadios I, 2 y 3 , que varían entre un año y medio y cuatro años, según cuál sea el elemento óseo considerado.

Las aves

Los huesos de aves pueden ser abundantes en los depósitos arqueológicos, especialmente en sectores costeros. Entre los taxones más representados se incluyen diseños anatómicos muy diferentes vinculados a diversos medios de locomoción, entre los que se destacan los pingüinos (Spheniscidae), aves buceadoras con una estructura corporal altamente especializada y sin huesos neumatizados. Estas diferencias osteológicas pueden ser factores que influyan en la resistencia de los huesos a la meteorización.

Los primeros estudios tafonómicos sobre huesos de aves fueron efectuados en Tierra del Fuego por Savanti y Muñoz (1998), quienes establecieron que meteorizan en una secuencia similar a la de los mamíferos y que desaparecen antes de alcanzar los estadios más altos. Estos aspectos fueron corroborados en Amboseli (Behrensmeyer et al. 2003) y en Patagonia continental (Cruz 2007a, 2007b, 20I I; entre otros).

Las observaciones actuales en el sur de Patagonia continental permitieron comparar la forma en que este proceso afecta los huesos de aves voladoras y de pingüinos (Cruz 2007a, 2009, 20I I). Como puede observarse en la Figura I, sus perfiles de meteorización son bastante similares, a pesar de las diferencias osteológicas.
El ñandú (Rhea pennata)

Las características anatómicas del ñandú y su peso de aproximadamente $27 \mathrm{~kg}$ lo separan de las otras aves patagónicas desde el punto de vista de su aprovechamiento y de la preservación de sus huesos. Las investigaciones desarrolladas por Belardi (1999) y Cruz (2007b, Cruz y Fernández 2004) en Patagonia continental permitieron establecer que existen marcadas diferencias entre elementos en relación a este proceso. Aunque algunos huesos de ñandú pueden meteorizar tan rápidamente como los de otras aves, los correspondientes a las extremidades posteriores meteorizan más lentamente, por lo que su preservación puede ser mayor a la del resto del esqueleto (Cruz 2007b, 20 I I).

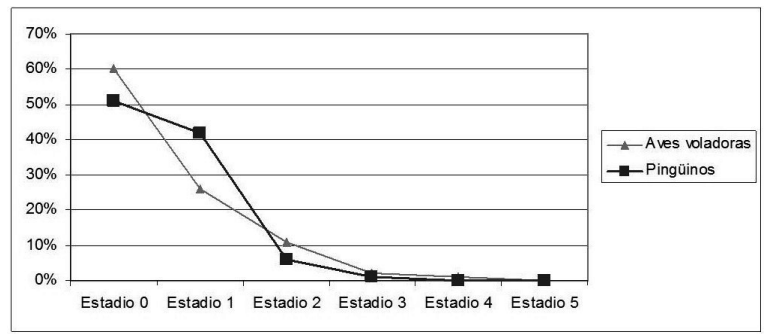

Figura 1: Comparación de los perfiles de meteorización de aves voladoras y pingüinos (Spheniscidae) en Patagonia Meridional, según datos de Cruz (2009).

Figure 1: Comparison of weathering profiles for flying bird and penguin (Spheniscidae) bones in Southern Patagonia, data from Cruz (2009)

\section{COMPARACIONES ENTRETAXONES}

La forma en que meteorizan los huesos de distintos taxones (Figura 2), particularmente sus tasas, pueden tener implicaciones importantes en lo que respecta a la representación taxonómica y anatómica, dos aspectos centrales del análisis zooarqueológico. Ya se señalaron las diferencias y similitudes en la meteorización de huesos de distintas aves patagónicas y entre elementos correspondientes a diferentes unidades anatómicas de los ñandúes. A continuación se presentan otras diferencias detectadas entre taxones. 


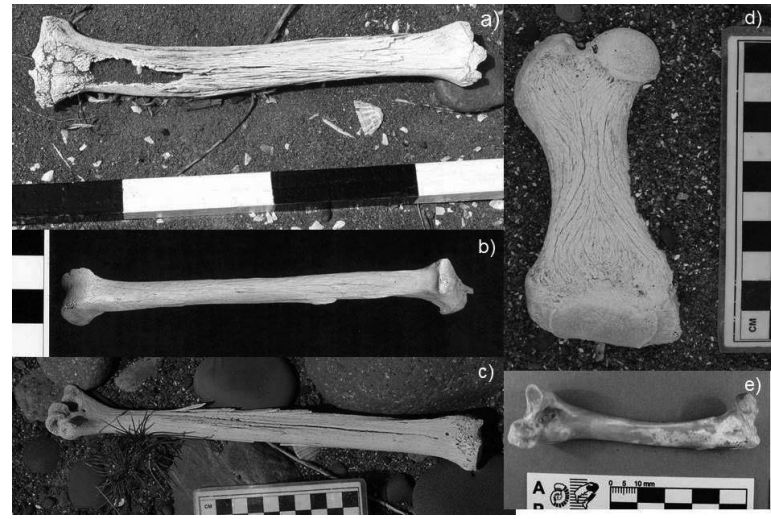

Figura 2: Estadios de meteorización en huesos de diferentes taxones: a) tibia de guanaco en estadio 4 ; b) tibiatarso de pingüino de Magallanes en estadio 3; c) tarsometatarso de ñandú patagónico en estadio 4; d) fémur de lobo marino en estadio 3; e) fémur de ave voladora (Chloephaga picta) en estadio 0 .

Figure 2: Weathering stages for bones of different taxa; a) guanaco tibia in stage 4; b) Magellanic penguin tibiatarsus in stage 3; c) Lesser Rhea tarsometatarsus in stage 4; d) South American sea lion femur in stage 3; e) flying bird (Chloephaga picta) in stage 0

En Patagonia los huesos de mamíferos tienen tasas de meteorización más lentas que los de aves (Cruz 2007b, 2008, 20 I I). Los huesos actuales de aves presentan perfiles en los que predominan los huesos no meteorizados 0 levemente meteorizados y en los que son escasos los estadios más altos, lo cual indica una rápida destrucción por este proceso. En los mismos contextos, los huesos de mamíferos muestran una supervivencia más prolongada a través de perfiles de meteorización que incluyen todos los estadios, incluso los más altos (Cruz 2007b, 2008). Este es un patrón similar al establecido en la sabana africana (Behrensmeyer et al 2003).

Los huesos de mamíferos pequeños, como los cánidos, meteorizan más rápidamente que los de guanacos (Cruz y Muñoz 2010), mientras que los huesos de pinnípedos meteorizan más lentamente que los de guanacos en un mismo contexto ambiental (Cruz y Muñoz 20ll). Los huesos de guanaco y algunos de ñandú meteorizan más lentamente que los de aves voladoras y pingüinos, por lo que sus posibilidades de conservarse en los conjuntos de superficie, de ser cubiertos por sedimentos y, por lo tanto, de formar parte de conjuntos arqueológicos, son mayores (Cruz 2009).

\section{DISCUSIÓNY CONCLUSIONES}

Las investigaciones presentadas abarcan una serie de aspectos, entre los que se destaca la consideración del impacto de la meteorización sobre huesos de cada especie sin suponer similitudes con la propuesta de Behrensmeyer. Un punto de importancia es que se registraron diferencias con la secuencia establecida en Amboseli, como en el caso de los cetáceos (Borella 2004) y los pinnípedos, aunque para estos últimos todavía sea necesario describir las particularidades del patrón para cada estadio. Las características particulares de la estructura ósea de estos mamíferos marinos puede ser un factor de importancia para que la secuencia de modificaciones que produce la meteorización en los huesos de mamíferos terrestres sea diferente, aspecto que habrá que monitorear en estudios futuros.

También se detectaron variaciones en las tasas de meteorización, vinculadas a las características climáticas y ambientales propias de la región (Borrero 2007). Es importante que se haya comenzado a considerar la necesidad de establecer las tasas relativas para diferentes taxones en un mismo contexto (Cruz 2007b, 2008; Cruz y Muñoz 20l0). Las investigaciones en Patagonia no sólo confirmaron la existencia de diferencias entre taxones, sino también entre huesos con diferentes grados de fusión y entre elementos de una misma carcasa (Borella 2004, Borrero 2007, Cruz 2007b, 2009, 201I; Cruz y Muñoz 2010). Si bien no pueden aplicarse directamente al registro zooarqueológico debido a que dependen de factores climáticos y ambientales locales, las tasas pueden ser consideradas como una medida relativa para evaluar la integridad de los conjuntos zooarqueológicos. Un ejemplo para Patagonia (Cruz 2007b): dada su mayor resistencia, la presencia en un depósito de huesos de guanaco con un perfil de meteorización que incluya los estadios más altos indica que no hay garantía de la preservación de los huesos de otros taxones que pudieran haberse depositado inicialmente. Inversamente, si un depósito enterrado incluye huesos poco meteorizados de los taxones más frágiles, como las aves, es posible plantear que las condiciones de preservación son buenas y que se habrán conservado gran parte de los huesos. 
Como en toda investigación arqueológica, la interpretación de las propiedades de los conjuntos óseos debe efectuarse en el marco de principios y modelos generales, en este caso relativos a la preservación y destrucción de restos orgánicos. Por eso, las investigaciones sobre meteorización no necesitan ser diseñadas ad hoc para resolver problemas de un depósito particular, sino que merecen ser desarrolladas para construir este marco general que permitirá comprender los casos específicos.

Por último, subrayo la necesidad de aplicar los resultados en el análisis de conjuntos arqueológicos específicos. Una evaluación crítica en diferentes contextos permitiría avanzar en la generación de principios más ajustados sobre la meteorización $y$, por lo tanto, más apropiados para la interpretación zooarqueológica en este sector del continente.

\section{AGRADECIMIENTOS:}

Este es un resultado de los proyectos UNPA 29/A302 y PIP/CONICET I I 2-20080I-00996.

\section{BIBLIOGRAFÍA}

Behrensmeyer, A.K. 1978. "Taphonomic and Ecological Information from Bone Weathering". Paleobiology 4:150-162.

Behrensmeyer, A.K., C.T. Stayton y R.E. Chapman 2003. "Taphonomy and Ecology of Modern Avifaunal Remains from Amboseli Park, Kenya”. Paleobiology 29:52-70.

Belardi, J.B. 1999. "Hay choiques en la terraza. Información tafonómica y primeras implicaciones arqueofaunísticas para Patagonia”. Arqueología 9:163-185.

Borella, F. 2004. Tafonomía Regional y Estudios Arqueofaunísticos de Cetáceos en Tierra del Fuego y Patagonia Meridional. BAR International Series 1257, Oxford.

Borella, F. y A.S. Muñoz 2006. "Observaciones tafonómicas sobre restos de pinnípedos en la costa norte fueguina (Argentina)". Intersecciones en Antropología 7:399. 403.

Borrero, L. 1990. "Taphonomy of Guanaco Bones in Tierra del Fuego". Quaternary Research 34:36I-37I.

---- 2000. "Ten Years After: esquema para una tafonomía regional de la Patagonia Meridional y norte de Tierra del Fuego". En Desde el país de los gigantes. Perspectivas arqueológicas en Patagonia, editado por J.B. Belardi, F. Carballo Marina y S. Espinosa, Tomo I:183-193. UNPA, Río Gallegos.

Borrero, L.A. 2007. "Longitudinal Taphonomic
Studies in Tierra del Fuego, Argentina". En Taphonomy and Archaeozoology in Argentina, editado por M.A. Gutiérrez, L. Miotti, G. Barrientos, G.o Mengoni Goñalons y M. Salemme, Pp. 219-233. BAR International Series SI60I, Oxford.

Cruz, I. 2007a. "Avian Taphonomy: Observations at Two Magellanic Penguin (Spheniscus magellanicus) Breeding Colonies and Their Implications for the Fossil Record". Journal of Archaeological Science 34:1252-I26I.

----2007b. “The recent bones of the Río Gallegos Basin (Santa Cruz, Argentina) and their preservation potential". En Taphonomy and Archaeozoology in Argentina, editado por M.A. Gutierrez, L. Miotti, G. Barrientos, G. Mengoni Goñalons y M. Salemme, Pp. 161-170. BAR International Series SI60I, Oxford.

..--2 2008."Avian and Mammalian Bone Taphonomy in Southern Continental Patagonia. A Comparative Approach". Quaternary International 180:30-37.

-.--2009."Tafonomía en escalas espaciales amplias: el registro óseo de las aves en el sur de Patagonia". Temas de Arqueología 2: 15-34.

---2 201 I."Tafonomía de huesos de aves. Estado de la cuestión y perspectivas desde el sur del Neotrópico". Antípoda 13:147-174.

Cruz, I. y P.M. Fernández 2004.“Comprendiendo el pasado: avances en tafonomía de Rheidos". Actas del $1^{\circ}$ Congreso Latinoamericano sobre Conservación y Cría Comercial de Ñandúes. INTA-Secretaría de Agricultura, Ganadería, Pesca y Alimentación, Argentina.

Cruz, I. y A.S. Muñoz 2010. "Tafonomía comparativa: seguimiento de carcasas de mamíferos en Punta Entrada (Santa Cruz, Argentina)". En Zooarqueología a principios del siglo XXI: aportes teóricos, metodológicos y casos de estudio, editado por M.A. Gutiérrez, M. De Nigris, P. M. Fernández, M. Giardina, A. F. Gil, A. Izeta, G. Neme y H. D. Yacobaccio, pp. 387-396. Ediciones del Espinillo, Buenos Aires.

-.--20II.“Meteorización de restos óseos de vertebrados: cambio y estabilidad en carcasas y conjuntos actuales en Punta Entrada y Monte León (costa atlántica de Patagonia)". Trabajo presentado en II Congreso Nacional de Zooarqueología Argentina, Olavarría, Argentina.

Lyman, R.L. 1994. Vertebrate Taphonomy. Cambridge University Press, Cambridge.

Muñoz, A.S. y F. Savanti 1998. "Observaciones tafonómicas sobre restos avifaunísticos de la costa noreste de Tierra del Fuego". Actas y Memorias del XI Congreso Nacional de Arqueología Argentina ( $8^{a}$ Parte), Revista del Museo de Historia Natural de San Rafael Tomo XX 1/2:107-121.

Tappen, M. 1994. "Bone Weathering in the Tropical Rain Forest".Journal of Archaeological Science 21:667-673. 